\title{
Evolution of the latitudinal diversity gradient in the hyperdiverse ant genus Pheidole
}

$+$

Evan P. Economo ${ }^{1,2^{*}}$, Jen-Pan Huang ${ }^{2}$, Georg Fischer ${ }^{1}$, Eli M. Sarnat ${ }^{1}$, Nitish Narula ${ }^{1}$, Milan Janda $^{3,4}$, Benoit Guénard ${ }^{5}$, John T. Longino ${ }^{6}$, L. Lacey Knowles ${ }^{2}$

*correspondence: Evan P. Economo, evaneconomo@gmail.com

${ }^{1}$ Okinawa Institute of Science and Technology Graduate University, Onna, Okinawa, Japan, 904-0495

${ }^{2}$ Department of Ecology \& Evolutionary Biology, Museum of Zoology, University of Michigan, USA

${ }^{3}$ National Laboratory for Ecological Analysis and Synthesis (LANASE), ENES, UNAM, Morelia, Mexico

${ }^{4}$ Biology Centre of Czech Academy of Sciences, Ceske Budejovice, Czech Republic

${ }^{5}$ The University of Hong Kong, School of Biological Sciences, Hong Kong, SAR, China.

${ }^{6}$ Department of Biology, University of Utah, USA

Acknowledgements: This work was supported by NSF (DEB-1145989 to EPE and LLK), by subsidy funding to OIST, and by a Japan Society for the Promotion of Science Kakenhi (17K15180) to EPE. We thank P.S. Ward and B.L. Fisher for sharing specimens and for all the data contributors to the GABI project.

Biosketch: The research team is interested in the ecology and evolution of biodiversity, This is the author manuscript accepted for publication and has undergone full peer review but has not been through the copyediting, typesetting, pagination and proofreading process, which may lead to differences between this version and the Version of Record. Please cite this article as doi: $10.1111 /$ geb.12867

This article is protected by copyright. All rights reserved 
especially insects.

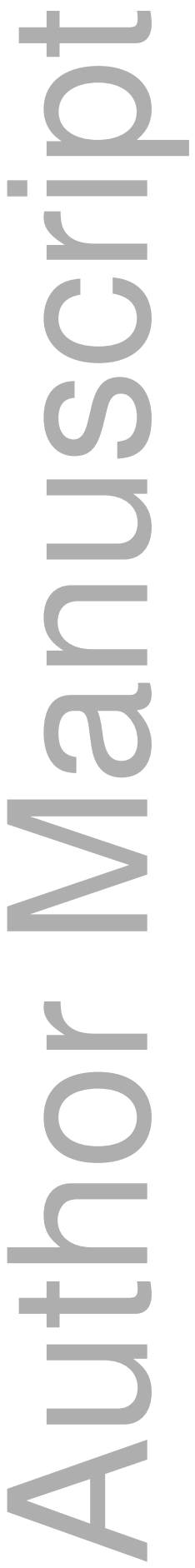

This article is protected by copyright. All rights reserved 
2 PROFESSOR EVAN ECONOMO (Orcid ID : 0000-0001-7402-0432)

3 DR. BENOIT GUENARD (Orcid ID : 0000-0002-7144-1175)

4 DR. JOHN T. LONGINO (Orcid ID : 0000-0001-5465-0293)

5

6

7

8

9

10 1

Article type : Research Papers

\title{
Evolution of the latitudinal diversity gradient in the hyperdiverse ant genus Pheidole
}

\begin{abstract}
Running title: Evolution of tropical hyperdiversity in ants
\end{abstract}
Evan P. Economo ${ }^{1,2 *}$, Jen-Pan Huang ${ }^{2}$, Georg Fischer ${ }^{1}$, Eli M. Sarnat ${ }^{1}$, Nitish Narula ${ }^{1}$, Milan Janda $^{3,4}$, Benoit Guénard ${ }^{5}$, John T. Longino ${ }^{6}$, L. Lacey Knowles ${ }^{2}$

*correspondence: Evan P. Economo, evaneconomo@gmail.com

${ }^{1}$ Okinawa Institute of Science and Technology Graduate University, Onna, Okinawa, Japan, 904-0495

${ }^{2}$ Department of Ecology \& Evolutionary Biology, Museum of Zoology, University of Michigan, USA

${ }^{3}$ National Laboratory for Ecological Analysis and Synthesis (LANASE), ENES, UNAM, Morelia, Mexico

${ }^{4}$ Biology Centre of Czech Academy of Sciences, Ceske Budejovice, Czech Republic ${ }^{5}$ The University of Hong Kong, School of Biological Sciences, Hong Kong, SAR, China.

${ }^{6}$ Department of Biology, University of Utah, USA 


\section{ABSTRACT}

Aim

The latitudinal diversity gradient is the dominant pattern of life on Earth, but a consensus understanding of its origins has remained elusive. The analysis of recently diverged, hyperrich invertebrate groups provides an opportunity to investigate latitudinal patterns with the statistical power of large trees while minimizing potentially confounding variation in ecology and history. Here, we synthesize global phylogenetic and macroecological data on a hyperdiverse (>1100 species) ant radiation, Pheidole, and test predictions of three general explanations for the latitudinal gradient: variation in diversification rates, tropical conservatism, and ecological regulation.

\section{Location}

Global.

\section{Time Period}

The past 35 million years.

\section{Major taxa studied}

The hyperdiverse ant genus Pheidole Westwood.

\section{Methods}

We assembled geographic data for 1499 species and morphospecies, and inferred a dated phylogeny for 449 species of the Pheidole, including 167 species newly sequenced for this study. We tested for correlations between diversification rate and latitude with BAMM, HiSSE, GeoSSE, and FiSSE, evaluated evidence for richness steady state, and examined patterns of diversification as Pheidole spread around the globe.

61 Results

62 There was no evidence of systematic variation of net diversification rates with latitude across any of the methods. We found that Pheidole diversification occurred in bursts when new continents were colonized, followed by a slowdown in each region, but there is no evidence richness has saturated at an equilibrium in any region. Additionally, we found latitudinal 
affinity is moderately conserved with a Neotropical ancestor and simulations show that phylogenetic inertia alone is sufficient to produce the gradient pattern.

\section{Main Conclusions}

Our results provide no evidence that diversification rates vary systematically with latitude.

Richness is far from steady state in each region, contrary to the ecological regulation hypothesis, although there is evidence that ecological opportunity promotes diversification after colonization of new areas. The fact that niche conservatism is strong enough to produce the gradient pattern is in accord with the tropical conservatism hypothesis. Overall, these results shed light on the mechanisms underlying the emergence of the diversity gradient within the past 34 million years, complementing recent work on deeper timescales, and more generally contribute toward a much-needed invertebrate perspective on global biodiversity dynamics.

Keywords: ants, latitudinal diversity gradient, tropical conservatism, diversification rate, diversity regulation, macroevolution

82

\section{INTRODUCTION}

Understanding how ecological and evolutionary processes interact with historical factors to shape global biodiversity patterns remains a major goal of biology. The latitudinal diversity gradient (LDG) is the most general biogeographic pattern, yet we still lack a consensus understanding of its mechanisms (Pianka, 1966; Willig et al., 2003; Mittelbach et al., 2007; Fine, 2015). This is likely because many biological, physical, and historical factors that could plausibly affect diversity vary systematically with latitude, and thus a large number of hypotheses have been developed to explain the pattern. However, testing the predictions of different hypotheses empirically and evaluating their relative merits has proven to be a challenge.

Recently, the synthesis of large-scale geographic datasets along with large-scale phylogenetic data has provided new opportunities for empirical evaluation of hypotheses for 
the mechanisms underlying the LDG. These tests have mainly focused on vertebrates (e.g. Cardillo et al., 2005; Weir \& Schluter, 2007; Jetz et al., 2012; Pyron \& Wiens, 2013; Rolland et al., 2014; Duchêne \& Cardillo, 2015; Siqueira et al. 2016; Pigot et al., 2016) and woody plants (Kerkhoff et al., 2014), since those are the taxa with large-scale comprehensive data available. Several pioneering studies have examined latitudinal diversification patterns in insects (e.g. McKenna \& Farrell, 2006; Hawkins \& DeVries, 2009; Condamine et al., 2012; Moreau \& Bell, 2013; Pie, 2016; Owens et al., 2017), although data-deficiency of most invertebrate groups makes taxonomic and/or geographic scope a challenge for analysis.

Among invertebrates, ants are emerging as an exemplar taxon for global biodiversity studies. Ants are ecologically dominant in most terrestrial ecosystems and are, for an insect group, relatively well documented scientifically. Moreover, their diversity is high, but not intractably so, with richness on the same order as major vertebrate groups ( 15,000 described ant species), and ants exhibit a marked latitudinal diversity gradient (Kaspari et al., 2004; Dunn et al., 2009). Recently, a new comprehensive dataset has been compiled which gives the known geographic distribution of all described ant species across $>400$ geographic regions around the globe (Guénard et al., 2017). These data, combined with progress toward reconstructing the ant tree of life (Brady et al., 2006; Moreau et al., 2006; Moreau \& Bell, 2013; Ward et al., 2015), allow for inferences of the evolutionary underpinnings of largescale diversity patterns in ants.

Here, we use the globally distributed, hyperdiverse (>1100 described species) ant genus Pheidole as a model taxon to test hypotheses for the latitudinal diversity gradient. While over a hundred hypotheses have been proposed to explain the gradient (Willig et al., 2003; Mittelbach et al., 2007; Fine, 2015), these can be placed under three umbrella hypotheses: i) the Diversification Rate hypothesis (DRH), ii) the Tropical Conservatism Hypothesis (TCH), and iii) the Ecological Regulation Hypothesis (ERH).

The Diversification Rate Hypothesis posits that there is some causal factor that affects speciation and/or extinction rates and varies with latitude (e.g. reviewed in Pianka, 1966; Mittelbach et al.,2007; Fine, 2015). This leads to a latitudinal disparity in species accumulation rate that underlies the gradient, rather than any regulation of total species numbers. Many such potential causal factors have been proposed. For example, temperature may affect mutation rates, which in turn could affect the rates of evolution of reproductive incompatibilities (Rohde, 1992). Or, extinction rates could be higher in the temperate than tropical zone to due greater climatic variability (Weir \& Schluter, 2007; Rolland et al., 2014). 
132 The prediction of the DRH is straightforward: net diversification rate inferred from a phylogeny should be higher in tropical lineages compared with extratropical lineages. 2004) posits that the relative youth of colder temperate biomes combined with the inertia of

136 niche evolution (phylogenetic niche conservatism: Wiens \& Graham, 2005; Losos, 2008) has

137 limited the accumulation of diversity in the temperate zone. In this scenario, net

138 diversification rates or equilibrium levels do not necessarily vary with latitude, and the difference in richness is mainly due to time for diversification (Stephens \& Wiens, 2003).

140 This idea is based on the fact that historically the Earth has been much warmer than it is now, 141 and much of what is now the temperate zone was covered by "megathermal" biomes. This

142 hypothesis is supported by the fossil record; many lineages that used to occur in the

143 Palearctic are now limited to tropical latitudes. This is true for ants as well; the Baltic amber 144 ant fauna from the late Eocene has greater affinity to modern Indo-Australian faunas than 145 modern Palaearctic faunas (Guénard et al., 2015). The main prediction of this hypothesis is 146 that the ancestral region of most groups is the tropics (or areas with what we recognize now 147 as "tropical" climates that may have previously extended out of the tropics), transitions out of 148 the tropical zone are rare, and thus the temperate clades are younger and nested within 149 tropical clades. Transitions from tropical to temperate zones should be difficult because of 150 the many nontrivial adaptations that ectothermic organisms need to survive at higher 151 latitudes. An additional prediction of the $\mathrm{TCH}$ is that the accumulation of lineages in the 152 temperate zone through dispersal or cladogenesis has mostly occurred after the Oligocene cooling, 34mya.

154 The Ecological Regulation Hypothesis (ERH) posits that, due to causal factors that 155 vary with latitude, more species can coexist locally and regionally in tropical ecosystems than 156 in temperate ecosystems. In this case, diversity is saturated at or near an ecological limit, and 157 this "carrying capacity" of species varies with latitude. Equilibrium diversity levels may be 158 regulated by factors such as productivity, perhaps mediated through competition and limiting 159 similarity (Pianka, 1966; Hurlbert \& Stegen, 2014b; Rabosky \& Hurlbert, 2015). Speciation 160 and extinction rates may vary over time to regulate richness at the requisite equilibrium level 161 for a geographic region, but a latitudinal factor acting on speciation/extinction is not causally 162 responsible for the disparity in diversity. Likewise, latitudinal affinity may be highly 163 conserved or evolve quickly, but it would be immaterial to the origins of the gradient if 164 diversity is saturated and regulated at levels that vary with latitude. 
These hypotheses have been tested across broad taxonomic scales (e.g. birds: Jetz et al., 2012, amphibians: Pyron \& Wiens 2013; mammals: Buckley \& Jetz, 2007; Rolland et al., 2014), and predictions of the DRH and TCH have been recently examined on the scale of all ants using a comprehensive geographic dataset (Economo et al. 2018; also see Moreau \& Bell 2013; Pie 2016). That study found that tropical lineages are more ancient than extratropical lineages, which mainly arose since the Oligocene cooling (past 34my), consistent with the TCH. Further, they found that diversification rate was highly heterogeneous but uncorrelated with latitude among ant clades, inconsistent with the DRH. Due to the limitations of phylogenetic data at such broad taxonomic and time scales, they could not test for ecological regulation (ERH), As with other studies on broad taxonomic scales, the analyses across all ants provided the statistical advantages of big datasets and a deep-time perspective. However, deep-time analyses also can be confounded by the fact that many ecological, functional trait, and historical factors may affect macroevolutionary rates in ways that could obscure underlying latitudinal effects. For example, ant diversification rates have been shown to be heterogeneous across clades (Pie \& Tschá, 2009; Moreau \& Bell, 2013) and possibly related to functional traits (Blanchard \& Moreau, 2017). Analyses of closely related lineages allow us to control to some extent for differences in biology unrelated to the variable of interest (in this case latitudinal affinity), while the large trees of hyperdiverse radiations enhance statistical power to detect trait-dependent diversification (Gamisch, 2016). Moreover, latitudinal gradients are often present within individual clades that evolved recently (Economo et al., 2015a), and different ecological and evolutionary dynamics can dominate at different phylogenetic scales (Graham et al., 2018). Studies across broad phylogenetic/time scales may miss the relevant scale of variation in diversification rate. Thus, the analysis of closely related lineages within younger, hyper-rich radiations provides an important complement to studies of larger taxa and longer time scales.

The global radiation of Pheidole arose entirely since the Oligocene cooling (last 34my), during which time it has evolved a latitudinal gradient echoing the pattern for all ants (Economo et al., 2015a). Thus, Pheidole presents an opportunity to examine diversification dynamics in this most recent period since the Oligocene, a period when many ant lineages transitioned out of the tropics. While the low number of older extratropical ant lineages is consistent with the TCH (Economo et al., 2018), there is still an open question of whether niche conservatism, diversification rate differences, or ecological saturation explain the emergence of the gradient since the Oligocene. According to the $\mathrm{TCH}$, the tropical ancestry of Pheidole combined with a low rate of evolution in latitudinal affinity (i.e. high 
phylogenetic niche conservatism) explains why there are more species in the tropics in the absence of latitudinal differences in macroevolutionary rates or regulation at different levels. The DRH predicts that Pheidole is diversifying more rapidly in the tropics, and this explains the current observed disparity in richness. The ERH posits that diversity is saturated at different levels across latitudes, and thus we should see a period of high net diversification rates early in the radiation followed by declines to near zero as richness reaches steady state or "carrying capacity". The ERH is thus an equilibrium hypothesis while the DRH and TCH are both non-equilibrium hypotheses.

Here, we reconstruct a new global Pheidole phylogeny-the most comprehensive to date-increasing substantially the taxonomic and geographic coverage from previous studies of the genus (Moreau, 2008; Sarnat \& Moreau, 2011; Economo et al., 2015a; Economo et al., $2015 b)$. We use the new phylogeny and geographic data from the GABI database to test predictions of the three umbrella hypotheses for the latitudinal gradient. As mechanisms involved with different hypotheses can be simultaneously operating (for example, speciation rate can vary with latitude even while niche conservatism limits colonization of the extratropics), our goal is to rule out mechanisms rather than isolate a single exclusive answer.

215 The analysis of this famously hyperdiverse radiation will advance our general understanding

216 of the latitudinal gradient, the most pervasive pattern of life on Earth.

\section{METHODS}

\section{Geographic Data}

221 Our geographic data are based primarily on the Global Ant Biodiversity Informatics Project

222 (GABI) database (Guénard et al., 2017) which can be viewed through the website

223 antmaps.org (Janicki et al., 2016), and secondarily on the personal collection records of the

224 authors (all of which are available on AntWeb.org). The former focuses on described species, 225 while the second was used to supplement data on morphospecies for taxa included in the 226 phylogenetic analysis. Because many records of ant occurrence are not associated with 227 geocoordinates, we assigned each record to a system of 415 polygons around the world

228 (Figure 1). Latitudinal range for a species was estimated as minimum and maximum 229 latitudes over all polygons in which a species occurs, excluding occurrences where the 230 species is considered exotic and dubious records. For statistical analyses, we used either the 231 absolute midpoint latitude of the range, an index of tropicality (fraction of latitudinal range in the tropics - fraction of latitudinal range outside the tropics, with $+/-23.5^{\circ}$ latitude as the 
boundary of the tropics). For tests using a binary coding latitudinal state, we used midpoint

234 latitude within or outside $+/-23.5^{\circ}$ to separate tropical and extratropical taxa.

\section{Phylogeny reconstruction}

237 Taxon Selection: Compared with many other large ant radiations, the effort to reconstruct the 238 phylogenetic history of Pheidole is relatively far along. A series of studies, beginning with 239 Moreau (2008) and followed by others (Sarnat \& Moreau, 2011; Economo et al., 2015a;

240 Economo et al., 2015b) has produced a broad picture of the evolutionary history of the genus.

241 However, for the purposes of understanding geographic patterns of diversification, having a

242 larger, and more proportionally sampled phylogeny will provide additional statistical power

243 and more robust results. Thus, we continued sampling Pheidole taxa for sequencing, focusing 244 on sampling more taxa from the Neotropics, Madagascar, and SE Asia, which had been 245 undersampled in previous analyses. In all, we increased the number of species from 282 taxa 246 in the most recent global Pheidole phylogeny (Economo et al., 2015a) to 449 taxa in the 247 current contribution (Table S2).

249 Estimation of Sampling Completeness: One source of uncertainty in large-scale analyses of 250 diversity is bias in taxonomic completeness overall and among different areas, particularly in relatively poorly known groups such as insects. While there is still a pronounced latitudinal gradient in Pheidole even among described species, there are undoubtedly many undescribed species in the genus, and it is probable they are disproportionately found in the tropics. While accounting for unobserved species is a challenge in any analysis, we devised an approximate method to calculate sampling completeness across areas given the information available, and use these estimates in our analysis of diversification rate. The details of our calculation are in the Supporting Information.

DNA Sequencing: Previous molecular work (Moreau 2008, Sarnat \& Moreau 2011, Economo et al. 2015a) on Pheidole has generated a dataset based on eight nuclear loci [His3.3B (histone H3.3B F1 copy), Lop1 (long wavelength sensitive opsin 1), GRIK2

262 (glutamate receptor ionotropic, kainate 2-like), unc_4 (unc-4 homeodomain gene), LOC15 263 (uncharacterized locus LOC15), CAD (carbomoylphosphate synthase), EF-1 $\alpha$ F2 (elongation 264 factor 1-alpha F2), Top1 (DNA topoisomerase 1)], and one mitochondrial locus [CO1

265 (cytochrome oxidase 1)]. In a previous study (Economo et al. 2015a), all 9 loci were 
sequenced for a subset of 65 taxa representing the main Pheidole lineages around the world, while three loci (COI, Lop1, and His3.3B) were sequenced for all taxa to fill out the clades (217 taxa). This hierarchically redundant sampling design was chosen for reasons of cost and time efficiency and to maximize the number of taxa, combined with the fact that many of the slow-evolving nuclear genes provide less information on recent divergences.

We added 167 new Pheidole taxa to this existing dataset by extending this sampling design and sequencing COI, Lop1, and His3.3B. We did not plan to sequence all 9 loci unless we found novel divergent clades not represented by taxa with all 9 genes sequenced in the earlier study (and we did not). Ant samples from field collections fixed in 95\% EtOH were extracted for DNAs using DNeasy Blood \& Tissue Kit (Qiagen, Hilden, Germany). The whole ant body was incubated in the extraction buffer without grinding during the first step, and then the complete ant specimen was removed before filtering and cleaning the extracts via a provided column. Extracted DNAs were subsequently used for PCR reactions for one mitochondrial (CO1) (Folmer et al., 1994) and two nuclear (His3.3B and Lop1) regions. Each reaction contained $0.5 \mathrm{ul}$ of extracted DNA, $1 \mathrm{ul}$ of $10 \times$ buffer, $0.75 \mathrm{ul}$ of $\mathrm{MgCl} 2,0.5 \mathrm{ul}$ of $10 \mathrm{mN}$ dNTPs, $0.2 \mathrm{ul}$ of $1 \%$ BSA, $0.4 \mathrm{ul}$ of of each primer, $0.04 \mathrm{ul}$ of Tag DNA polymerase (Invitrogen, USA), and $\mathrm{ddH}_{2} \mathrm{O}$ to make a total of $10 \mathrm{ul}$ reaction. Standard PCR procedures were employed with annealing temperatures of 52, 60, and $60 \mathrm{C}$ for CO1, His3.3B, and Lop1 regions, respectively. The amplicons were sequenced via a $\mathrm{ABI}^{3700}$ machine by the Sequencing Core at the University of Michigan. Sequences were checked using SeqMan (DNAStar Inc., USA).

Phylogenetic tree inference: We used Bayesian methods to infer a dated Pheidole phylogeny including 449 ingroup taxa (Table S2). To generate codon-aware alignments for these loci, we first searched NCBI's non-redundant CDS database (Clark et al., 2016) for reliable amino acid sequences for all loci and retrieved such sequences for seven of the nine loci with the following accession numbers: AIM2284.1 (CAD), ABW70333.1(CO1), EZA53539.1 (EF-1 $\alpha$ F2), EGI60526.1(His3.3B), ABW36758.1 (Lop1), EGI59282.1 (unc-4), and AIM43286.1 (Top1). These sequences were used as references for generating codon-aware alignments. The CAD, unc-4, and Top1 alignments generated using MAFFT v7.205 (Katoh \& Standley, 2013) (--retree 4; --maxiterate 1000) showed no frameshift mutations and/or insertions and deletions. However, the CO1, EF-1 $\alpha$ F2, His3.3B, and Lop1 alignments did not match the reference sequences, showing disruptions in the translated amino acid alignments (such as the presence of numerous stop codons). For these loci, we used a codon-aware alignment 
300

301

302

303

304

305

306

307

308

309

310

311

312

313

314

315

316

317

318

319

320

321

322

323

324

325

326

327

328

329

330

331

332

333

software, MACSE v1.01b (Ranwez et al., 2011), to generate the alignments. Reverse translations of the reliable amino acid reference sequences, accounting for all possibilities at each codon position, were passed as reliable input sequences to the software, we were able to assign codon positions within the exons in these seven loci. The resulting alignments were manually inspected and cleaned using Geneious R8 software. Furthermore, we identified, extracted, and separately aligned intronic regions wherever necessary. The remaining two loci, LOC15 and GRIK-2, were aligned using MAFFT. We concatenated all nine alignments and once again manually cleaned the master alignment, resulting in an alignment containing 8839 sites.

We used PartitionFinder v1.1.1 (Lanfear et al., 2012) to determine the partitioning scheme and corresponding models of molecular evolution. The model scope included HKY, $\mathrm{HKY}+\Gamma, \mathrm{SYM}, \mathrm{SYM}+\Gamma, \mathrm{GTR}, \mathrm{GTR}+\Gamma, \operatorname{TrN}, \operatorname{TrN}+\Gamma, \mathrm{K} 80, \mathrm{~K} 80+\Gamma, \operatorname{TrNef}, \operatorname{TrNef}+\Gamma, \mathrm{JC}$, and $\mathrm{JC}+\Gamma$, branch lengths were set to 'linked', and model selection and comparison was set to Bayesian Information Criterion (BIC). PartitionFinder identified an optimal scheme containing 16 partitions (Table S3). We used ClockstaR (Duchêne et al., 2014) to determine the optimal number of clock models across our partitions for relaxed-clock phylogenetics, and a single linked clock was preferred based on the SEMmax criterion.

Our primary phylogenetic inference was conducted in BEAST2 v2.1.3 (Bouckaert et al., 2014), but we first performed maximum likelihood (ML) reconstruction in RAxML v8.0.25 (Stamatakis, 2014). Using the partitioning scheme described above and the GTR $+\Gamma$ model, we ran $75 \mathrm{ML}$ inferences with 1000 bootstraps to find the ML tree. Using the chronos function in the ape package in R (Paradis et al., 2004), we scaled the tree by calibrating the root node to a range of 50-60my. This tree was used as the starting tree for the BEAST2 analyses, but the topology was not fixed.

Unfortunately there are no reliable fossil calibrations available to date nodes within the genus. Thus, the age of the group can only be informed by the age of the stem node and information from fossils in related taxa across the subfamily Myrmicinae. Because our analysis is concentrated within Pheidole, we preferred to use the stem node age distribution (i.e. the most recent common ancestor of Pheidole and its sister lineage Cephalotes+Procryptocerus) inferred as in a much larger analysis of the subfamily Myrmicinae (Ward et al., 2015) that could make use of a broad range of molecular and fossil data. Following those results, the stem node calibration was set to a normal distribution (mean: 58.0 mya, sigma, 4.8my). Further analysis of the Pheidole fossil record with a goal to place fossil taxa within the Pheidole phylogeny and refine dating of different nodes in the 
tree, represents an important need for future phylogenetic work on the genus. Despite this limitation, the analyses in this paper depend mostly on relative - rather than absolute - ages, and we draw no conclusions based on the precise timing of nodes in the tree.

We used a relaxed lognormal clock model linked across partitions (due to the ClockstaR results), and used the partitioning scheme and models identified with PartitionFinder. Six independent analyses were run and chains were stopped between 45 and 80 million generations, after we observed convergence using Tracer software v1.6.0 (Rambaut 2014). We discarded the leading 33\% of saved states as burnin, combined the remaining trees from all runs to create the posterior set, and generated the Maximum Clade

343 Credibility tree and nodes set to median height. After pruning the outgroup, this tree was used 344 for all subsequent analyses.

\section{Macroevolutionary rate inference}

We took several complimentary approaches to estimating macroevolutionary rates and potential dependencies on latitude, primarily basing our anlaysis on BAMM (Bayesian Analysis of Macroevolutionary Mixtures, Rabosky, 2014), HiSSE (Hidden State Speciation and Extinction, Beaulieu \& O'Meara, 2016), and FiSSE (Rabosky \& Goldberg, 2017), with a secondary analysis using GeoSSE (Geographic State Speciation and Extinction, Goldberg et al., 2011). These methods each have their strengths and weaknesses thus our approach is to use them collectively to seek conclusions about our data that are robust to methodological assumptions and implementation.

The main advantage of BAMM is that complex mixture models can be assessed with rate shifts across the tree, including potentially accelerating and/or decelerating diversification rates. While trait-dependent diversification models are not fit directly, traitdiversification correlations can be assessed post hoc using structured rate permutations that estimate correlations while accounting for phylogenetic dependency (Rabosky \& Huang, 2015). We use BAMM to test for correlations between latitude and net diversification rate, and evaluate evidence of decelerating diversification to a steady state (ecological regulation of diversity) overall and in relation to the colonization of continents.

While BAMM has strengths in inferring complex mixtures of diversification processes, they are not explicitly trait-dependent, and the SSE family of methods explicitly fits models of trait-dependent diversification. SSE methods have been developed with different kinds of trait data, either based on binary traits (BISSE, Maddison et al., 2007), continuous traits (QuaSSE, FitzJohn, 2010), or explicitly geographic traits (GeoSSE, 
368 Goldberg et al., 2011). While these methods are explicitly for inferring trait-dependent speciation and extinction, they have the problem that differences in the focal trait are the only mechanisms that can cause shifts in macroevolutionary rates. If the real process has complex rate shifts then a more complex trait dependent model may fit better than a homogeneous null model, even if the shifts are not related to the traits per se, leading to type-I errors (Rabosky \& Goldberg, 2015). These problems are at least partially solved by HiSSE (Beaulieu \& O'Meara, 2016), a method that fits binary trait-dependent speciation and extinction models that can be formally tested against similarly complex trait-independent models. We thus primarily used HiSSE for our analysis. Since GeoSSE has been implemented for explicitly geographic dynamics, we also fit that model as a secondary test and present that analysis in the supplement.

Finally, as an additional test for variation in speciation rate with latitude, we used a non-parametric method, FiSSE (Rabosky \& Goldberg, 2017), that does not depend on an assumed model structure and is robust to false inferences of trait-dependent evolution given a range of underlying complex evolutionary dynamics. FiSSE is limited to testing speciation rate differences; it does not directly test for net diversification rate differences. However, many (but not all) hypotheses for why diversification rate could vary with latitude are based on mechanisms acting on speciation rate, so it is a partial test of the broader Diversification Rate Hypothesis.

BAMM implementation: We estimated net-diversification, speciation, and extinction rates through time for the inferred Pheidole tree using the program BAMM V2.5. The initial values for speciation rate, rate shift, and extinction rate were estimated using the setBAMMpriors function from the R package BAMMtools (Rabosky et al., 2014). Specifically, a total of $2 \times 10^{8}$ generations of rjMCMC searches with samples stored every 8000 generations were performed using speciation-extinction. A total of 1000 post burnin samples $(50 \%)$ were retained. We performed two BAMM runs for each of three assumptions about sampling completeness (L, M, H) accounted for by changing the GlobalSamplingFraction parameter $(0.3,0.22,0.18$, respectively, see Supplemental Information for justification). To account for potential oversampling of Nearctic species, we performed a series of runs where we lowered the number of Nearctic species by randomly pruning 21 (of total 48) Nearctic tips from the tree ten times and performed a BAMM run on each replicate, using the $\mathrm{M}$ assumption for the GlobalSamplingFraction parameter. 

overall pattern of Pheidole diversification, 2) assess whether there is evidence of diversity regulation, particularly decelerating diversification to zero over time and after colonization of new areas, and 3) test for latitudinal dependency in diversification rate while accounting for phylogenetic non-independence. We visualized the lineage specific diversification with the plot.bammdata function from BAMMtools, and the time plot of clade-specific diversification rate was plotted with the plotRateThroughTime function. We used STRAPP (e.g. the traitDependentBAMM function in BAMMtools) to test for significance of any latitudediversification correlations. We tested for diversification rate vs. either tropicality index or absolute midpoint latitude (one-tailed, 10000 iterations, Spearman's rho as test statistic). We also checked whether our results were robust to using Pearson correlation as test statistic or coding latitude as a binary variable (tropicality $>0$ or tropicality $<0$ ) and using Mann-Whitney test.

HiSSE Implementation: The HiSSE approach (Beaulieu \& O'Meara, 2016) extends the BiSSE (Binary State Speciation and Extinction model) (Maddison et al., 2007) framework with two advances. First, the HiSSE model itself allows for more complex models in which macroevolutionary rates can be the function of the focal trait and a hidden state. Thus, if our focal character has states 1 and 0 (in our case tropical and extratropical), there could be an influence of a second unobserved character (with states A and B) on a macroevolutionary rates $\lambda$ and $\mu\left(\lambda_{0 \mathrm{~A}}, \lambda_{0 \mathrm{~B}}, \lambda_{1 \mathrm{~A}}, \lambda_{1 \mathrm{~B}}\right)$. Second, importantly, it allows the fitting of null characterindependent models (CID) in which a hidden factor(s) underlies diversification rate changes without the influence of the focal trait under investigation. This allows trait-dependent BiSSE models to be compared to a character-independent model of similar complexity (CID-2, with two hidden states A and B) and more complicated HiSSE models to be compared to models of similar complexity (CID-4, with four hidden states A, B, C, D). BiSSE (trait dependent speciation-extinction), HiSSE (trait-dependent speciation/extinction with hidden states that also affect speciation/extinction) and CID (trait-independent models with hidden states that affect speciation/extinction) are best used together and models with all structures can be compared.

We fit a range of models with increasing complexity, starting with the BiSSE family of models under the following sets of constraints on the parameters: all diversification and transition rates equal among states, diversification equal but transition rates different (i.e. 
speciation and or extinction changes with latitude, but transition rates among temperate and tropical are equal), diversification different but transition rates equal (i.e. speciation and extinction vary with state, but transition rates are equal), or all rates free unconstrained to vary with state (the full BiSSE model).

The HiSSE models allow speciation/extinction/transition rates to vary with the focal trait and also among two hidden traits. One question in implementing HiSSE is how to set the transition parameters among states (combination of observed 0/1 and hidden A/B states, with combined state space $0 \mathrm{~A}, 1 \mathrm{~A}, 0 \mathrm{~B}, 1 \mathrm{~B})$. We followed suggestions of the authors of the method (Beaulieu \& O'Meara, 2016), either setting all transition rates to be equal, or assumed a three parameter rates in which transitions between the observed states could vary but transitions between hidden states is a single parameter. The CID-2 and CID-4 models are fit including 2 or 4 hidden states, respectively, but with no dependence on the observed traits. For these, we also assumed alternatively a single rate for all state transitions (observed and hidden) or a three-rate model including two rates for transitions between the observed states and one between all hidden states.

We implemented all of the above analyses using functions in the $\mathrm{R}$ package hisse (Beaulieu \& O'Meara, 2016). As with the BAMM analysis, we ran all models using either the (L, M, H) assumptions about sampling completeness, and analyzed both the global Pheidole and New World only. For the New World analyses, we additionally adjusted the sampling fraction $\left(\mathrm{M}^{*}\right)$ to account for possible undersampling of tropical species relative to extratropical species. As the ML optimization does not always find the global minimum from a single starting point, we ran $20 \mathrm{ML}$ searches for each model using random starting parameters chosen from a uniform distribution on the interval $(0,1)$. For all the models above, we ran them alternatively assuming a fixed root in the tropical state, or root probability estimated with the default "madwitz" method based on the data. As we found the results were insensitive to the root method, we only present results with the default option. After all BiSSE, HiSSE, and CID models were inferred, we compared all models with sample-size corrected Akaike's Information Criterion (AICc) scores.

FiSSE Approach: FiSSE (Rabosky \& Goldberg, 2017) is a nonparametric test for traitdependent speciation rates that does not assume an underlying model structure, but rather depends on distributions of branch lengths in the different states. FiSSE is complementary to the BiSSE and is robust to Type-I error. We performed both one-tailed and two-tailed tests of 
global Pheidole and only the New World Pheidole. We also performed FiSSE on a set of trees for the New World only where temperate species were thinned to account for possible undersampling of the tropics (see Supporting Information).

Phylogenetic niche conservatism: While previous studies have shown that Pheidole likely has a tropical ancestor (Moreau 2008, Economo et al. 2015a), it remains an open question whether phylogenetic niche conservatism is strong enough to produce a gradient pattern during the Oligocene period, a key predictions of the TCH. We performed analyses to evaluate the degree to which latitudinal affinity is phylogenetically conserved in Pheidole, and used simulations to test if that conservation is strong enough for a gradient to emerge given tropical ancestry alone. For this, we first calculated two measures of phylogenetic signal-Blomberg's K (Blomberg et al., 2003) and Pagel's lambda (Pagel, 1999) — treating absolute latitudinal midpoint as a continuous trait, using the phylosig() function in the $\mathrm{R}$ package phytools (Revell, 2012). Second, to estimate the overall evolutionary rates, we fit models of discrete character evolution (treating latitudinal affinity as a binary variable) using the fitDiscrete() function in the R package geiger (Pennell et al., 2014). To visualize the evolution of latitudinal affinity, we performed 100 stochastic character maps on the empirical tree using the make.simmap() function, and plotted a summary of state probabilities with the function densityMap(), both from the phytools package. Finally, to estimate whether the inferred rate of evolution combined with tropical ancestral state is consistent with the observed richness difference even in the absence of diversity regulation and diversification rate differences, we simulated niche evolution on the empirical tree and maximum likelihood model with the sim.history() function from phytools. While tree shape and trait state are not necessarily independent (i.e. the dependent model is implemented in the BiSSE/HiSSE analyses), this analysis asks whether we would be likely to observe a gradient even if they were independent, given that Pheidole likely has a tropical ancestor and given the rate that latitudinal affinity evolves. Pheidole likely has a tropical ancestor as Pheidole fimbriata, which is sister to the rest of Pheidole, and the sister lineage of Pheidole, Cephalotes + Procryptocerus, are all tropical (Moreau, 2008; Ward et al., 2015).

\section{RESULTS}

Pheidole exhibits a latitudinal diversity gradient that is overall similar to ants as a whole (Fig. 1). The BEAST analysis inferred a phylogeny whose major features are consistent with previous studies (Figs. 2, S1). The crown age of the group (i.e. the mrca of 
503 Pheidole fimbriata with the rest of Pheidole) is inferred here to be younger than in a previous study ( 29mya vs. 37mya in Economo et al., 2015a).

According to the BAMM analysis, the hyperdiversification of Pheidole began after an acceleration approximately 15-16 mya, and all species except for two early-diverging lineages (P. fimbriata and P. rhea) are descended from this event (Figure 2). Diversification initially occurred in the New World, exhibiting a decelerating trend over time. Around 13mya, a single lineage colonized the Old World and this was associated with another burst of diversification followed by a slowdown in a clade encompassing Asia and Africa. Madagascar and Australia-NG were later colonized, followed by accelerations and

512 subsequent decelerations in each clade (Figs. 2, S1, S2). There were several other

513 accelerations that were not obviously associated with geographic transitions, including one

514 clade in the New World and the megacephala group in the Afrotropics. This general pattern

515 of sequential colonization-acceleration-deceleration pattern is robust to changing the sampling fraction parameter, although as one would expect, the inferred degree of deceleration becomes less pronounced if one assumes that more species are left to be sampled. However, there is no evidence that diversity is saturated (i.e. net diversification rate approaching zero) as net diversification rates were all strongly positive (ranging between 0.20.5 across regions and analyses with different parameter assumptions).

The extratropical lineages generally belong to young clades nested within larger tropical clades (Figs. 2, S1). While diversification rates vary across the genus to a degree, we could not detect a significant correlation (assessed with STRAPP) between BAMM-inferred net diversification rate and either absolute midpoint latitude or tropicality index across any of the analyses we performed (Fig. 3). These results were similar across variation in the assumed global sampling fractions, whether we calculated correlations for individual clades or the whole tree, and including trees where Nearctic species were culled to account for possible uneven sampling. Although significance tests were one-tailed for higher diversification in the tropics, we also note that none of the observed correlation coefficients were outside the null range in either direction.

The HiSSE analysis was also broadly consistent with BAMM analysis in finding no statistical support for a correlation between macroevolutionary rates and latitude. In general,

533 the CID-2 trait-independent null model outperformed the BiSSE trait-dependent models, and 534 the CID-4 null outperformed the HiSSE trait-dependent models, and the CID-4 models had 535 the global minimum AICc across the different permutations of the analysis (Table 1). Thus, 536 this analysis provided no evidence for latitude-dependent macroevolution in this genus. It is 
worth noting as well that the AIC-minimizing versions of the BiSSE and HiSSE models, which again were themselves not preferred over the null models, generally did not support higher diversification rate in the tropics. The BiSSE model detected a slightly higher

540 diversification rate in the extratropical zone and the HiSSE model either fit models where

541 tropical diversification was higher than extratropical while in one hidden state and lower in

542 the other hidden state, or where the extratropical diversification was always higher in both

543 hidden states. For the New World, use of the sampling effort correction removed this slight,

544 and non-significant difference. The GeoSSE analysis showed overall similar results to

545 BiSSE, with a positive latitude-diversification rate trend in the New World, but not global,

546 Pheidole; however, the association is not robust to the correction for latitudinal

547 undersampling (see supplement).

548 The FiSSE analysis was also consistent with the other analyses in showing no

549 correlation between speciation rate and latitude for global Pheidole $\left(\lambda_{\text {temp }}=0.28, \lambda_{\text {trop }}=0.27\right.$,

550 two tailed $\mathrm{p}>0.88$ ), but did show a positive speciation-latitude correlation for the New World

551 alone $\left(\lambda_{\text {temp }}=0.30, \lambda_{\text {trop }}=0.20\right.$, two-tailed $\left.\mathrm{p}<0.026\right)$. However, when we dropped extratropical

552 tips from the phylogeny to simulate potential latitudinal undersampling of the tropics, this

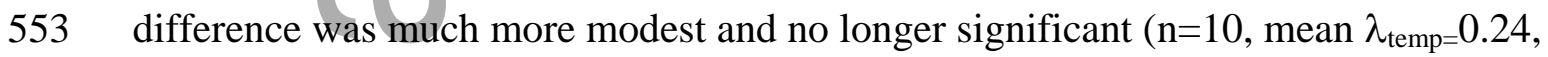

554 S.E. $=0.005$, mean $\lambda_{\text {trop }}=0.20$, S.E. $=0.0002$, p range: $0.19-0.72$ among replicates).

555 The extratropical lineages are clustered with each other on the tree, although it is clear 556 there were numerous transitions out of the tropics (Fig. 4). The tests for phylogenetic signal 557 in latitudinal affinity for Blomberg's $K(K=0.34, \mathrm{p}<0.002)$ and Pagel's lambda $(\lambda=0.95$, $558 \mathrm{p}<10^{-57}$ ) were both highly significant. Symmetric and asymmetric models of discrete 559 character evolution both fit the data comparably well (symmetric model $\mathrm{q}_{\text {trop-> etrop }}=\mathrm{q}_{\text {etrop- }}$ $560>$ trop $=0.015, \mathrm{AICc}=235.5$, asymmetric model $\mathrm{q}_{\text {trop }>\text { etrop }}=0.013, \mathrm{q}_{\text {etrop }>\text { trop }}=0.060$,

561 AICc=234.9). Simulations of character evolution on the empirical phylogeny show that a

562 latitudinal gradient is the most common outcome if one assumes a tropical ancestor and either model for the inferred rate of evolution of latitudinal affinity (Fig. 4).

\section{DISCUSSION}

Our analysis of Pheidole macroevolution sheds light on the mechanisms responsible 567 for the evolution of the latitudinal diversity gradient in ants. By focusing on the dynamics of 568 a massive radiation in the post-Oligocene, our study complements taxon-wide studies that 569 focus on differences among highly divergent clades and deeper timescales (e.g. Cardillo et 
We find no evidence of higher diversification rate for tropical Pheidole lineages across any of our analyses (Figs. 2-4, S1), as would be predicted by the Diversification Rate

574 Hypothesis. In general, the signal of latitude as a trait affecting macroevolutionary rates in

575 the BAMM, HiSSE, and FiSSE analyses was weak to non-existent. When there was some hint of a correlation, for example in the best fitting (but still not better than null) HiSSE/BiSSE analyses, and the FiSSE analysis for New World speciation rate uncorrected for latitudinal sampling bias, it was in the direction of higher diversification/speciation in the temperate zone. However, those correlations were not robust to reasonable assumptions about undersampling in the tropics, thus the overall picture is a lack of evidence for latitudinal dependency for macroevolutionary rates.

We do not view our analysis as ruling out that such systematic macroevolutionlatitude relationships may exist, even in Pheidole. Rather, our analysis only suggests that such relationships are not the causal factor in the gradient. The Diversification Rate Hypothesis assumes that lineages reach different latitudes early on in their evolution, and the disparity of richness is due to different accumulation rates over time. If niche conservatism is too high for lineages to evolve out of the tropics (or vice versa) early on in the radiation, there may be no chance for any latitude-macroevolutionary rate correlations to manifest and be statistically detectible. Thus, we view our analysis as stronger evidence that a diversification rate-latitude correlation is not causal in the latitudinal gradient in Pheidole, rather than showing that no such relationship exists. The finding that a latitudinal gradient in diversification rates does not underlie the diversity gradient in Pheidole echoes similar results for birds (Weir \& Schluter, 2007; Jetz et al., 2012; Rabosky \& Huang, 2012), butterflies (Owens et al., 2017), marine fishes (Rabosky et al., 2018; but see Siquera et al., 2016).

595 However, other recent work on mammals (Rolland et al., 2014), amphibians (Pyron \& Wiens, 596 2013), and studies on the fossil record (e.g. Jablonski et al., 2006) that indicated elevated net 597 diversification rates in the tropics. Thus, there continues to be disagreement across studies

598 and taxonomic groups. Whether this reflects true process variation across clades or 599 differences in conceptual and methodological approaches across studies remains an open 600 question.

Contemporary net diversification rates are positive in Pheidole across all regions, 602 with current rates varying between 0.25-0.50 across regions and assumptions about missing 603 taxa. This contradicts a key proposition of the Ecological Regulation Hypothesis, that 

diversity is at an equilibrium "carrying capacity" across regions (Pianka, 1966; Hurlbert \& Stegen, 2014b; Rabosky \& Hurlbert, 2015). There is evidence, however, that ecological opportunity at least partially controls diversification rate in Pheidole. Specifically, each time a new continent is colonized, diversification initially increases followed by a slowdown as richness increases, which can be a sign of niche filling (but not necessarily, see discussions in Moen \& Morlon, 2014; Harmon \& Harrison 2015). This could indicate that diversity will eventually saturate at a steady state if net diversification rate continues to decrease (at current rates of rate decrease, this would occur in about 10-20 million years). However, diversity dependence is not in itself evidence of ecological limits (Harmon \& Harrison, 2015), and it is

613 equally plausible that richness would not saturate but instead reach a peak and then decline,

614 resulting in a pulse or boom-bust pattern for the clade (e.g. as envisaged by Ricklefs, 2014).

615 The further investigation of the role of ecological limitations on diversity, and any latitudinal 616 differences in those limits, remains an important direction for future work. One promising 617 direction from a completely different angle would be to examine how niche overlap and 618 coexistence in Pheidole varies with latitude or energetic constraints, as has been pursued in 619 better studied taxa such as birds (e.g. Pigot et al., 2016), but the ant data are not yet available 620 at high enough resolution to pursue such analyses.

Overall, the results match the predictions of the tropical conservatism hypothesis (TCH). We found that latitudinal affinity is moderately conserved in Pheidole. While there 623 have been a number of transitions from the tropics to the temperate zone, latitudinal affinity evolves slowly enough to make a richness gradient the most likely outcome simply due to tropical ancestry and phylogenetic inertia. Thus, our study joins a series of recent studies supporting the TCH for woody plants (Kerkhoff et al., 2014), birds (Duchêne \& Cardillo, 2015), mammals (Buckley et al., 2010), and butterflies (Hawkins \& DeVries, 2009). These results for Pheidole evolution in the post-Oligocene connect well to results on ant diversification on deeper timescales (Economo et al., 2018), and together tell a coherent 630 story about the evolution of latitudinal gradients in ants across scales. Most ant lineages older 631 than 34mya are reconstructed to be tropical, including the Pheidole stem lineage. Around 15 mya, Pheidole exhibited a many-fold acceleration in diversification rate and began a massive radiation. The reason for this initial acceleration, such as evolution of a key innovation, remains unknown. It took time for some Pheidole lineages to evolve the requisite traits for colonization of high latitudes. Once colonization of cold biomes occurred, diversification was not detectibly slower. In their analysis across all ant clades, Economo et al. (2018) also found no evidence for elevated net diversification rates among clades centered in the tropics relative 
to those in the temperate zone, although clades are quite heterogeneous in rate, probably due to other latent biological and historical differences. It remained possible that diversification

640 rate was correlated with latitude within the large clades, but biological differences among

641 clades obscured this pattern. Within Pheidole, diversification rate is much less

642 heterogeneous, but there is still no evidence of a negative latitudinal correlation, implying

643 that lack of phylogenetic resolution within large clades was not hiding this relationship in the 644 previous analysis (Economo et al. 2018). Further work is needed to unravel diversification 645 patterns of other hyperdiverse ant clades (e.g. Camponotus, Strumigenys, Tetramorium,

646 Crematogaster) that also exhibit strong latitudinal gradients to confirm this apparent

647 consistency across phylogenetic scales. Indeed, these five hyperdiverse genera (out of 334

648 total ant genera) constitute over a third of all ant described ant species ( 5000), so each has a 649 marked effect on ant-wide patterns such as latitudinal gradients.

650 While our results are most consistent with tropical niche conservatism (TCH)

651 explaining the Pheidole latitudinal gradient, other patterns such as the diversification pulses

652 after colonization of new regions cannot be explained by phylogenetic niche conservatism

653 alone. Rather, even as our study rejects the main assertion of the ERH that contemporary

654 richness is regulated in steady state-Pheidole richness is apparently far from equilibrium

655 everywhere - we find evidence for an underlying mechanism of the ERH that diversity and

656 diversification are regulated by ecological opportunity and the filling of niche space. This

657 underscores the point that the mechanisms underlying the broad umbrella hypotheses are not

658 mutually exclusive, and even though a particular mechanism may not be causal for the 659 gradient, it may still be an important process operating in the diversification of different 660 groups, or operate on different phylogenetic scales in the same group (Graham et al., 2018).

661 Further quantitative approaches may be necessary to disentangle different mechanisms

662 operating simultaneously. We agree with the approach advocated by some (Hurlbert \&

663 Stegen, 2014a) toward a quantitative formulation of multiple competing and intersecting 664 hypotheses, combined with a simulation-based approach to identify their key predictions. We 665 felt initial efforts in this direction were not yet mature enough to use as a basis for the current 666 study, but look forward to further development of the approach in the future.

Despite the high level of research effort directed toward understanding the latitudinal gradient, the matter is far from resolved (Mittelbach et al., 2007). Studies have differed in their conclusions about the origins of the gradient, probably due to both differences in conceptual and methodological approaches and real variation in process and history across taxonomic groups. The former should continue to improve as we develop more penetrating 
quantitative methods that make use of more diverse data types. Variability across taxonomic groups is best assessed and understood by examining more of them. With development of global invertebrate datasets like the one analyzed here, we stand to broaden our perspective on large-scale biological patterns and their origins.

676

677

\section{REFERENCES:}

679

Beaulieu, J.M. \& O'Meara, B.C. (2016) Detecting hidden diversification shifts in models of trait-dependent speciation and extinction. Systematic Biology, 65, 583-601.

Blanchard, B.D. \& Moreau, C.S. (2017) Defensive traits exhibit an evolutionary trade-off and drive diversification in ants. Evolution, 71, 315-328.

Blomberg, S.P., Garland, T. \& Ives, A.R. (2003) Testing for phylogenetic signal in comparative data: behavioral traits are more labile. Evolution, 57, 717-745.

Bouckaert, R., Heled, J., Kuhnert, D., Vaughan, T., Wu, C.H., Xie, D., Suchard, M.A., Rambaut, A. \& Drummond, A.J. (2014) BEAST 2: a software platform for Bayesian evolutionary analysis. PLoS Computational Biology, 10, e1003537.

Brady, S.G., Schultz, T.R., Fisher, B.L. \& Ward, P.S. (2006) Evaluating alternative

Buckley, L.B., Davies, T.J., Ackerly, D.D., Kraft, N.J.B., Harrison, S.P., Anacker, B.L., diversity gradient in mammals. Proceedings of the Royal Society B: Biological Sciences, 277, 2131-2138.

Cardillo, M., Orme, C.D.L. \& Owens, I.P. (2005) Testing for latitudinal bias in diversification rates: an example using New World birds. Ecology, 86, 2278-2287.

Clark, K., Karsch-Mizrachi, I., Lipman, D.J., Ostell, J. \& Sayers, E.W. (2016) GenBank. Nucleic Acids Research, 44, D67-D72.

Condamine, F.L., Sperling, F.A.H., Wahlberg, N., Rasplus, J.Y. \& Kergoat, G.J. (2012) What causes latitudinal gradients in species diversity? Evolutionary processes and 
ecological constraints on swallowtail biodiversity. Ecology Letters, 15, 267-277.

707

708

709

710

711

712

713

714

715

716

717

718

719

720

721

722

723

724

725

726

727

728

729

730

731

732

733

734

735

736

737

738

739

Duchêne, D.A. \& Cardillo, M. (2015) Phylogenetic patterns in the geographic distributions of birds support the tropical conservatism hypothesis. Global Ecology and Biogeography, 24, 1261-1268.

Duchêne, S., Molak, M. \& Ho, S.Y.W. (2014) ClockstaR: choosing the number of relaxedclock models in molecular phylogenetic analysis. Bioinformatics, 30, 1017-1019.

Dunn, R.R., Agosti, D., Andersen, A.N., Arnan, X., Bruhl, C.A., Cerdá, X., Ellison, A.M., Fisher, B.L., Fitzpatrick, M.C. \& Gibb, H. (2009) Climatic drivers of hemispheric asymmetry in global patterns of ant species richness. Ecology Letters, 12, 324-333.

Economo, E.P., Klimov, P., Sarnat, E.M., Guenard, B., Weiser, M.D., Lecroq, B. \& Knowles, L.L. (2015a) Global phylogenetic structure of the hyperdiverse ant genus Pheidole reveals the repeated evolution of macroecological patterns. Proceedings of the Royal Society B: Biological Sciences, 282, 20141416.

Economo, E.P., Sarnat, E.M., Janda, M., Clouse, R., Klimov, P.B., Fischer, G., Blanchard, B.D., Ramirez, L.N., Andersen, A.N., Berman, M., Guenard, B., Lucky, A., Rabeling, C., Wilson, E.O. \& Knowles, L.L. (2015b) Breaking out of biogeographical modules: range expansion and taxon cycles in the hyperdiverse ant genus Pheidole. Journal of Biogeography, 42, 2289-2301.

Economo, E.P., Narula, N., Friedman, N. R., Weiser, M. D., Guénard, B. (2018) Macroecology and macroevolution of the latitudinal diversity gradient in ants. Nature Communications, 9, 1778.

Fine, P.V.A. (2015) Ecological and evolutionary drivers of geographic variation in species diversity. Annual Review of Ecology, Evolution, and Systematics, 46, 369-392.

FitzJohn, R.G. (2010) Quantitative traits and diversification. Systematic Biology, 59, 619633.

Folmer, O., Black, M., Hoeh, W., Lutz, R. \& Vrijenhoek, R. (1994) DNA primers for amplification of mitochondrial cytochrome c oxidase subunit I from diverse metazoan invertebrates. Molecular Marine Biology and Biotechnology, 3, 294-299.

Gamisch, A. (2016) Notes on the statistical power of the Binary State Speciation and Extinction (BiSSE) Model. Evolutionary Bioinformatics Online, 12, 165-174.

Goldberg, E.E., Lancaster, L.T. \& Ree, R.H. (2011) Phylogenetic inference of reciprocal effects between geographic range evolution and diversification. Systematic Biology, 60, 451-465.

Graham, C.H., Storch, D., Machac, A. (2018) Phylogenetic scale in ecology and evolution. 
Guénard, B., Perrichot, V. \& Economo, E.P. (2015) Integration of global fossil and modern biodiversity data reveals dynamism and stasis in ant macroecological patterns. Journal of Biogeography, 42, 2302-2312.

Guénard, B., Weiser, M.D., Gomez, K., Narula, N. \& Economo, E.P. (2017) The Global Ant Biodiversity Informatics (GABI) database: synthesizing data on the geographic distribution of ant species (Hymenoptera: Formicidae). Myrmecological News, 24, 8389.

Harmon, L., \& Harrison, S. (2015) Species diversity is dynamic and unbounded at local and continental scales. American Naturalist, 185, 584-593.

Hawkins, B.A. \& DeVries, P.J. (2009) Tropical niche conservatism and the species richness gradient of North American butterflies. Journal of Biogeography, 36, 1698-1711.

Hurlbert, A.H. \& Stegen, J.C. (2014b) When should species richness be energy limited, and how would we know? Ecology Letters, 17, 401-413.

Jablonski, D., Roy, K., Valentine, J.W. (2006) Out of the tropics: evolutionary dynamics of the latitudinal diversity gradient. Science, 314, 102-106.

Janicki, J., Narula, N., Ziegler, M., Guenard, B. \& Economo, E.P. (2016) Visualizing and interacting with large-volume biodiversity data using client-server web-mapping applications: The design and implementation of antmaps.org. Ecological Informatics, 32, $185-193$.

Jetz, W., Thomas, G.H., Joy, J.B., Hartmann, K. \& Mooers, A.O. (2012) The global diversity of birds in space and time. Nature, 491, 444-448.

Kaspari, M., Ward, P.S. \& Yuan, M. (2004) Energy gradients and the geographic distribution of local ant diversity. Oecologia, 140, 407-413.

Katoh, K. \& Standley, D.M. (2013) MAFFT Multiple sequence alignment software version 7: Improvements in performance and usability. Molecular Biology and Evolution, 30, $772-780$.

Kerkhoff, A.J., Moriarty, P.E. \& Weiser, M.D. (2014) The latitudinal species richness gradient in New World woody angiosperms is consistent with the tropical conservatism hypothesis. Proceedings of the National Academy of Sciences of the United States of

773 Lanfear, R., Calcott, B., Ho, S.Y.W. \& Guindon, S. (2012) PartitionFinder: Combined 
selection of partitioning schemes and substitution models for phylogenetic analyses. Molecular Biology and Evolution, 29, 1695-1701.

Losos, J.B. (2008) Phylogenetic niche conservatism, phylogenetic signal and the relationship between phylogenetic relatedness and ecological similarity among species. Ecology Letters, 11, 995-1003.

Maddison, W.P., Midford, P.E. \& Otto, S.P. (2007) Estimating a binary character's effect on speciation and extinction. Systematic Biology, 56, 701-710.

McKenna, D.D. \& Farrell, B.D. (2006) Tropical forests are both evolutionary cradles and museums of leaf beetle diversity. Proceedings of the National Academy of Sciences, 103, 10947-10951.

Mittelbach, G.G., Schemske, D.W., Cornell, H.V., Allen, A.P., Brown, J.M., Bush, M.B., Harrison, S.P., Hurlbert, A.H., Knowlton, N. \& Lessios, H.A. (2007) Evolution and the latitudinal diversity gradient: speciation, extinction and biogeography. Ecology Letters, 10, 315-331.

Moen, D., Morlon, H. (2014) Why does diversification slow down? Trends in Ecology \& Evolution, 29, 190-197.

Moreau, C.S. (2008) Unraveling the evolutionary history of the hyperdiverse ant genus Pheidole (Hymenoptera : Formicidae). Molecular Phylogenetics and Evolution, 48, 224-239.

Moreau, C.S. \& Bell, C.D. (2013) Testing the museum versus cradle tropical biological diversity hypothesis: phylogeny, diversification, and ancestral biogeographic range evolution of the ants. Evolution, 67, 2240-2257.

Moreau, C.S., Bell, C.D., Vila, R., Archibald, S.B. \& Pierce, N.E. (2006) Phylogeny of the ants: diversification in the age of angiosperms. Science, 312, 101-104.

Owens, H.L., Lewis, D.S., Dupuis, J.R., Clamens, A.L., Sperling, F.A.H., Kawahara, A.Y., Guralnick, R.P. \& Condamine, F.L. (2017) The latitudinal diversity gradient in New World swallowtail butterflies is caused by contrasting patterns of out-of-and into-thetropics dispersal. Global Ecology and Biogeography, 26, 1447-1458.

Pagel, M. (1999) Inferring the historical patterns of biological evolution. Nature, 401, 877884.

Paradis, E., Claude, J. \& Strimmer, K. (2004) APE: Analyses of phylogenetics and evolution in R language. Bioinformatics, 20, 289-290.

Pennell, M.W., Eastman, J.M., Slater, G.J., Brown, J.W., Uyeda, J.C., FitzJohn, R.G., Alfaro, M.E. \& Harmon, L.J. (2014) geiger v2.0: an expanded suite of methods for fitting 
Pianka, E.R. (1966) Latitudinal gradients in species diversity - a review of concepts. American Naturalist, 100, 33-\&.

811 Pie, M.R. (2016) The macroevolution of climatic niches and its role in ant diversification. Ecological Entomology, 41, 301-307.

813 Pie, M.R., \& Tschá, M.K. (2009) The macroevolutionary dynamics of ant diversification. Evolution, 63, 3023-3030.

Pigot, A.L., Tobias, J.A. \& Jetz, W. (2016) Energetic constraints on species coexistence in birds. PLoS Biology, 14, e1002407.

817 Pyron, R.A. \& Wiens, J.J. (2013) Large-scale phylogenetic analyses reveal the causes of high tropical amphibian diversity. Proceedings of the Royal Society of London B: Biological Sciences, 280, 20131622.

Rabosky, D.L. \& Hurlbert, A.H. (2015) Species richness at continental scales is dominated by ecological limits. American Naturalist, 185, 572-583.

Rabosky, D.L. \& Goldberg, E.E. (2015) Model inadequacy and mistaken inferences of traitdependent speciation. Systematic Biology, 64, 340-355.

Rabosky, D.L. \& Huang, H. (2015) Minimal effects of latitude on present-day speciation rates in New World birds. Proceedings of the Royal Society of London B: Biological Sciences, 282, 20142889.

Rabosky, D.L. \& Goldberg, E.E. (2017) FiSSE: A simple nonparametric test for the effects of a binary character on lineage diversification rates. Evolution, 71, 1432-1442.

Rabosky, D.L., Grundler, M., Anderson, C., Shi, J.J., Brown, J.W., Huang, H. \& Larson, J.G. (2014) BAMMtools: an R package for the analysis of evolutionary dynamics on phylogenetic trees. Methods in Ecology and Evolution, 5, 701-707.

Rabosky, D.L., Chang, J., Title, P.O., Cowman, P.F., Sallan, L., Friedman, M., Kaschnner, K., Garilao, C., Near, T.J., Coll, M., Alfaro, M.E. (2018). An inverse latitudinal gradient in speciation rate for marine fish. Nature, 559, 392-395.

Ricklefs, R.E. (2014) Reconciling diversification: random pulse models of speciation and extinction. American Naturalist 184, 268-276.

Ranwez, V., Harispe, S., Delsuc, F. \& Douzery, E.J.P. (2011) MACSE: Multiple Alignment of Coding SEquences accounting for frameshifts and stop codons. Plos ONE, 6

841 Revell, L.J. (2012) phytools: an R package for phylogenetic comparative biology (and other 
843 Rohde, K. (1992) Latitudinal gradients in species diversity: the search for the primary cause. $844 \quad$ Oikos, 514-527.

845 Rolland, J., Condamine, F.L., Jiguet, F. \& Morlon, H. (2014) Faster speciation and reduced 846 extinction in the tropics contribute to the mammalian latitudinal diversity gradient. 847 PLoS Biology, 12, e1001775.

848 Santini, L., González-Suárez, M., Rondinini, C., Di Marco, M. (2017) Shifting baseline in 849 macroecology? Unravelling the influence of human impact on mammalian body mass. 850 Diversity and Distributions, 23, 640-659.

851 Sarnat, E.M. \& Moreau, C.S. (2011) Biogeography and morphological evolution in a Pacific 852 island ant radiation. Molecular Ecology, 20, 114-130.

853 Siqueira, A.C., Oliveira-Santos, L.G.R., Cowman, P.F., Floeter, S.R., Algar, A. (2016) Evolutionary processes underlying latitudinal differences in reef fish diversity. Global Ecology and Biogeography, 25, 1466-1476.

Stamatakis, A. (2014) RAxML version 8: a tool for phylogenetic analysis and post-analysis of large phylogenies. Bioinformatics, 30, 1312-1313.

Stephens, P.R. \& Wiens, J.J. (2003) Explaining species richness from continents to communities: The time-for-speciation effect in emydid turtles. American Naturalist, $161,112-128$.

Turvey, S.T. \& Fritz, S.A. (2011) The ghosts of mammals past: biological and geographical patterns of global mammalian extinction across the Holocene. Philosophical Transactions of the Royal Society of London B: Biological Sciences, 366, 2564-76.

Ward, P.S., Brady, S.G., Fisher, B.L. \& Schultz, T.R. (2015) The evolution of myrmicine ants: phylogeny and biogeography of a hyperdiverse ant clade (Hymenoptera: Formicidae). Systematic Entomology, 40, 61-81.

Weir, J.T. \& Schluter, D. (2007) The latitudinal gradient in recent speciation and extinction rates of birds and mammals. Science, 315, 1574-1576.

Wiens, J.J. \& Donoghue, M.J. (2004) Historical biogeography, ecology and species richness. Trends in Ecology \& Evolution, 19, 639-644.

Wiens, J.J. \& Graham, C.H. (2005) Niche conservatism: Integrating evolution, ecology, and conservation biology. Annual Review of Ecology Evolution and Systematics, 36, 519539.

Willig, M.R., Kaufman, D.M. \& Stevens, R.D. (2003) Latitudinal gradients of biodiversity: Pattern, process, scale, and synthesis. Annual Review of Ecology Evolution and 


\section{DATA ACCESSIBILITY}

879 Molecular sequences have been deposited to GenBank (see Table S1 for accession numbers).

880 We have also provided the alignment, BEAST xml file, and geographic dataset in a

881 supplemental data archive (Dryad XXX). The GABI dataset can be accessed on the 882 interactive website antmaps.org.

884 Acknowledgements: This work was supported by NSF (DEB-1145989 to EPE and LLK), by 885 subsidy funding to OIST, and by a Japan Society for the Promotion of Science Kakenhi 886 (17K15180) to EPE. We thank P.S. Ward and B.L. Fisher for sharing specimens and for all 887 the data contributors to the GABI project.

Biosketch: The research team is interested in the ecology and evolution of biodiversity, especially insects.

891

\section{Supplementary Information}

893

Appendix 1: Caleulation of sampling completeness across geography and clade

Appendix 2: GeoSSE methods, analysis, and discussion

Figure S1: An expanded version of the phylogeny depicted in Figure 2, with readable tips and node supports.

Figure Legends

907 Figure 1: Global patterns of Pheidole species richness plotted by a) geographic region and b)

908 5-degree latitudinal band for 1138 described species/subspecies and 361 morphospecies. For 909 comparison, latitudinal distribution of 13771 ant species excluding Pheidole are also 
910 depicted. Latitudinal richness is expressed as fraction of total richness (1499 for Pheidole,

91113771 for all other ants).

912

913 Figure 2: Diversification rate dynamics inferred with BAMM from a phylogeny of 449

914 Pheidole species. a) Median diversification rates through time of the major Pheidole clades.

915 The New World median excludes the two early branching species (P. rhea and P. fimbriata)

916 that fall outside the initial acceleration of Pheidole diversification. b) The maximum clade

917 credibility phylogeny colored with inferred net diversification rate. c) Latitudinal extent of all

918449 taxa included in the tree. A high-resolution version with taxon names visible is presented

919 in Figure S1. d) Probable locations of diversification rate shifts. Here, branch length is

920 proportional to probability of a shift.

921

922

923 Figure 3: Net diversification rate inferred with BAMM as a function of latitude.

924 Diversification rate of each Pheidole species (present day) inferred with BAMM using the

925 "M" assumption of sampling completeness per species a) as a function of latitudinal midpoint

926 and b) tropicality index, which varies from -1 for a species with a range located competely

927 outside the tropics to 1 for a species confined to the tropics. c) Spearman correlations (black

928 dots) for net diversification and either absolute midpoint latitude (left) or tropicality (right),

929 where the grey boxes reflect 95\% null distribution generated with STRAPP. L, M, H, reflect

930 different assumptions about unsampled species (low, medium, high estimates of total

931 numbers of Pheidole), while $\mathbf{M}^{*}$ are 10 trees where temperate species have been culled to

932 account for possible sampling bias (see methods).

933

934 Figure 4: Evolution of latitudinal affinity in Pheidole. a) Branch-wise probability of

935 ancestral tropical state inferred from stochastic character mapping. b-c) Histograms of

936 latitudinal richness differences between tropics and extratropics simulated with stochastic

937 character mapping on the empirical phylogeny assuming a tropical ancestor and the inferred

938 degree of niche conservation using symmetric (b) or asymmetric (c) models of character

939 evolution. The vertical dashed line is the empirical richness fraction.

940

This article is protected by copyright. All rights reserved 
Table 1: Summary of delta AICc from the BiSSE and HiSSE trait-dependent models, and the two null models, CID-2 and CID-4. CID-2 is similar in model complexity to the BiSSE model, while CID-4 is similar in model complexity to the HiSSE model. The models were run with different parameter constraints listed below. The $\mathrm{L}, \mathrm{M}, \mathrm{H}$, refer to the low, medium, and high estimates of missing taxa. $\mathrm{M}^{*}$ includes a correction for possible oversampling with latitude. The AICc minimizing model for each analysis is highlighted in bold.

\begin{tabular}{|c|c|c|c|c|c|c|c|c|}
\hline & 13 & \multicolumn{3}{|c|}{$\begin{array}{l}\text { Global Pheidole } \\
\text { ( } \triangle \mathrm{AICc})\end{array}$} & \multicolumn{4}{|c|}{$\begin{array}{l}\text { New World Pheidole } \\
\qquad(\triangle \mathrm{AICc})\end{array}$} \\
\hline Model & Description/constraint & $\mathrm{L}$ & M & $\mathrm{H}$ & $\mathrm{L}$ & M & $\mathrm{H}$ & $\mathbf{M}^{*}$ \\
\hline BiSSE & $\begin{array}{l}\text { Div. rates and transition rates } \\
\text { equal across latitudes }\end{array}$ & 69.9 & 67.4 & 69.6 & 18.3 & 23.2 & 27.8 & 23.2 \\
\hline BiSSE & $\begin{array}{l}\text { Div. rates equal, transition } \\
\text { rates vary with latitude }\end{array}$ & 69.4 & 66.9 & 69.2 & 19.4 & 24.2 & 28.9 & 22.3 \\
\hline BiSSE & $\begin{array}{l}\text { Div rates vary, transition rates } \\
\text { equal with latitude }\end{array}$ & 69.9 & 67.4 & 69.6 & 18.3 & 23.2 & 27.8 & 23.2 \\
\hline BiSSE & $\begin{array}{l}\text { Div. rates and transition rates } \\
\text { vary with latitude (full BiSSE } \\
\text { model) }\end{array}$ & 73.5 & 71.1 & 73.3 & 11.6 & 16.1 & 18.9 & 23.2 \\
\hline CID-2 null & $\begin{array}{l}2 \text { hidden states, } 1 \text { transition } \\
\text { parameter }\end{array}$ & 33.9 & 21.9 & 17.3 & 0.5 & 2.8 & 5.7 & 2.9 \\
\hline CID-2 null & $\begin{array}{l}2 \text { hidden states, } 3 \text { transition } \\
\text { parameters }\end{array}$ & 21.2 & 20.1 & 19.4 & 2.4 & 5.3 & 8.6 & 4.5 \\
\hline HiSSE & $\begin{array}{l}\text { Div rates vary with latitude } \\
\text { and two hidden states, } 1 \\
\text { transition parameter }\end{array}$ & 36.1 & 21.1 & 16.5 & 0.9 & 0.9 & 0.3 & 8.6 \\
\hline HiSSE & $\begin{array}{l}\text { Div rates vary with latitude } \\
\text { and two hidden states, } 3 \\
\text { transition parameters }\end{array}$ & 22.5 & 28.0 & 27.7 & 0.2 & 2.0 & 2.3 & 9.5 \\
\hline CID-4 null & $\begin{array}{l}\text { Div rates vary with four } \\
\text { hidden states, } 1 \text { transition }\end{array}$ & 22.5 & 11.1 & 4.6 & 1.6 & 0.0 & 0.0 & 0.0 \\
\hline CID-4 null & $\begin{array}{l}\text { Div. rates vary with four } \\
\text { hidden states, } 3 \text { transition } \\
\text { parameters }\end{array}$ & 0.0 & 0.0 & 0.0 & 0.0 & 1.1 & 0.4 & 5.7 \\
\hline
\end{tabular}


a)

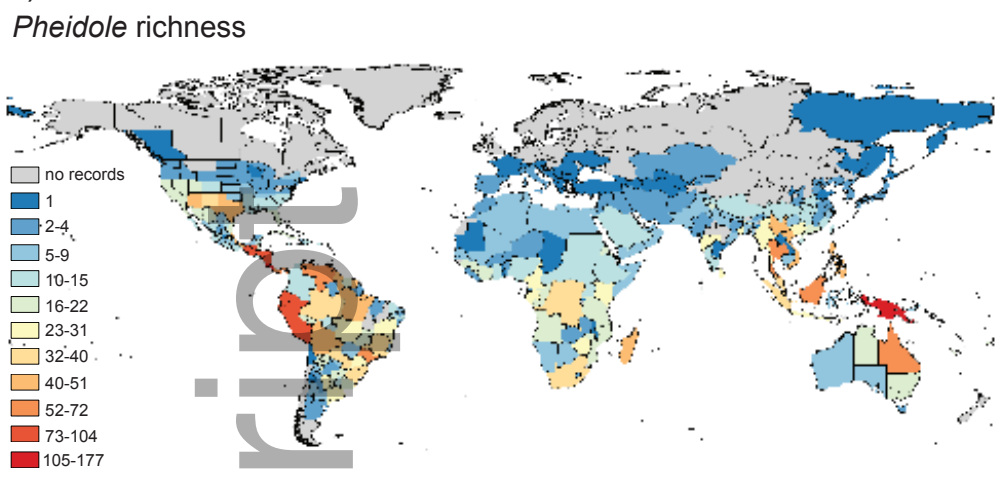

b)

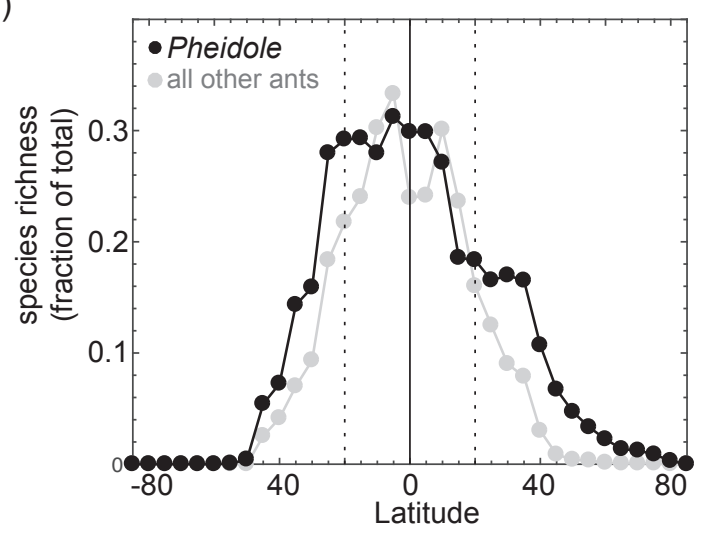

Figure 1 : Global patterns of Pheidole species richness plotted by a) geographic region and b) 5-degree latitudinal band for 1138 described species/subspecies and 361 morphospecies. For comparison, latitudinal distribution of 13771 ant species excluding Pheidole are also depicted. Latitudinal richness is expressed as fraction of total richness (1499 for Pheidole, 13,771 for all other ants).

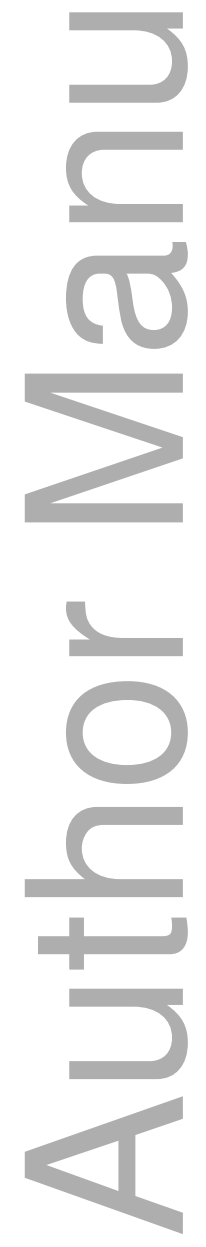




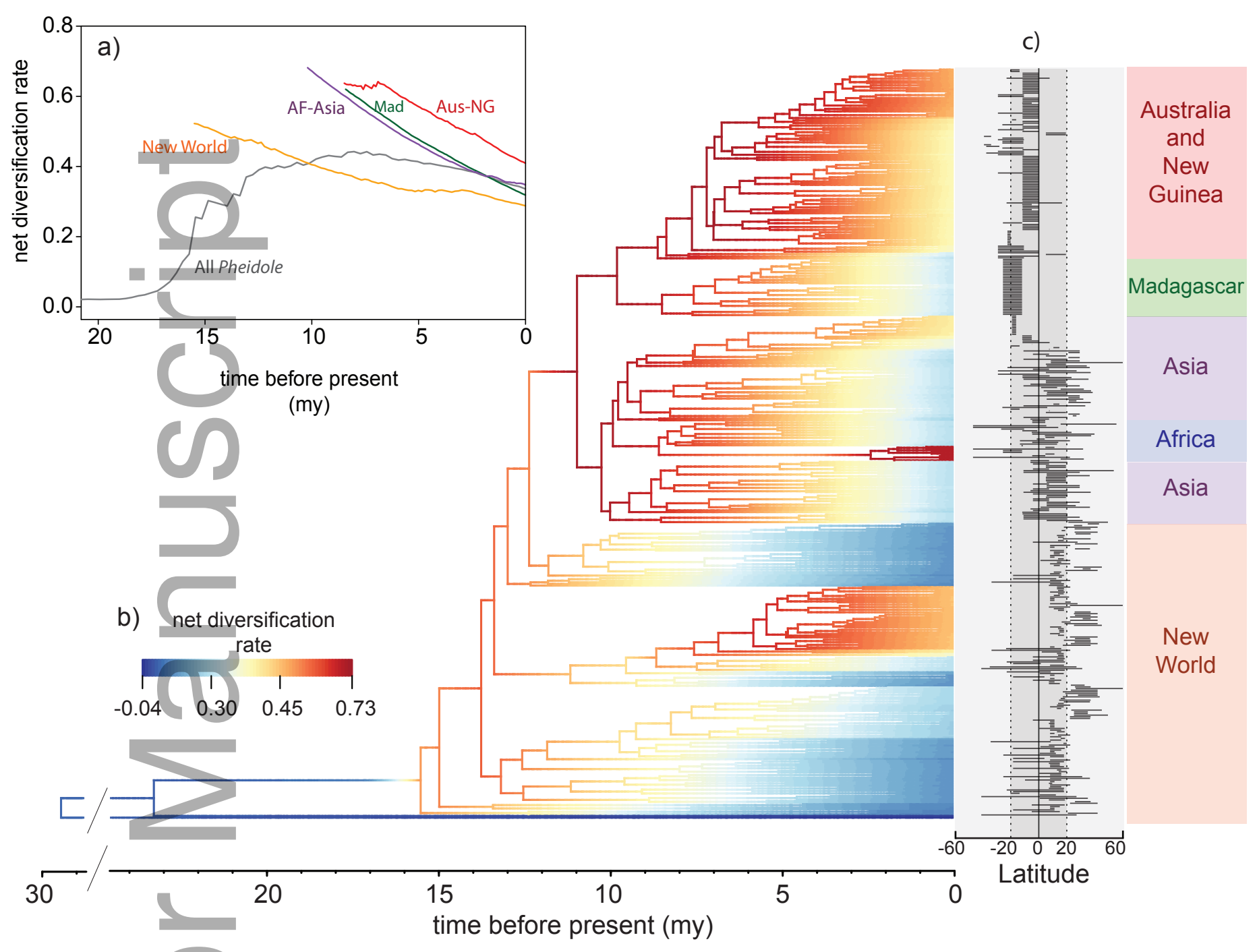

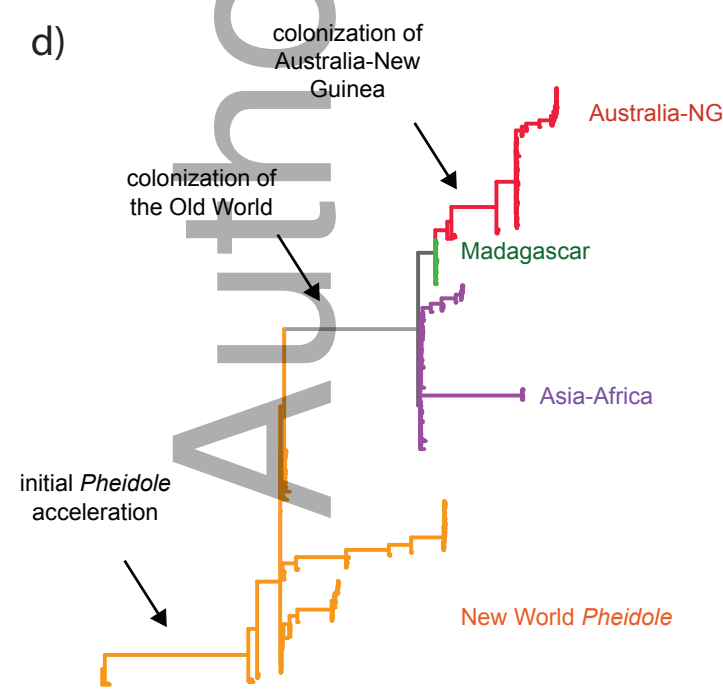

$\frac{\text { probability of rate shift }}{1.0}$
Figure 2: Diversification rate dynamics inferred with BAMM from a phylogeny of 449 Pheidole species. a) Median diversification rates through time of the major Pheidole clades. The New World median excludes the two basal species ( $P$. rhea and $P$. fimbriata) that fall outside the initial acceleration of Pheidole diversification. b) The maximum clade credibility phylogeny colored with inferred net diversification rate. c) Latitudinal extent of all 449 taxa included in the tree. A high-resolution version with taxon names visible is presented in Figure S1. d) Probable locations of diversification rate shifts. Here, branch length is proportional to probability of a shift. 
a)
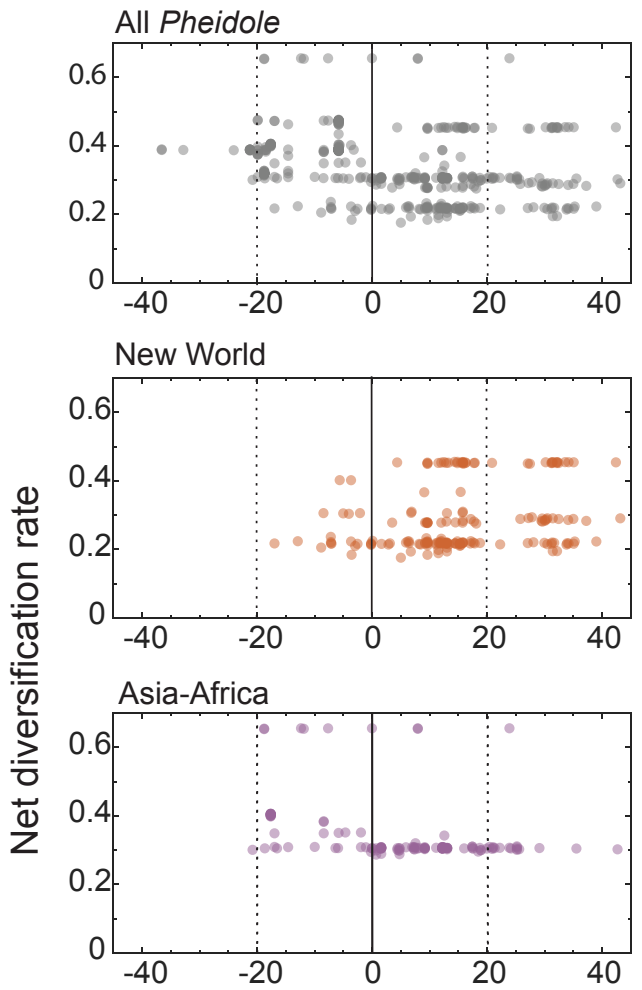

Aus-NG

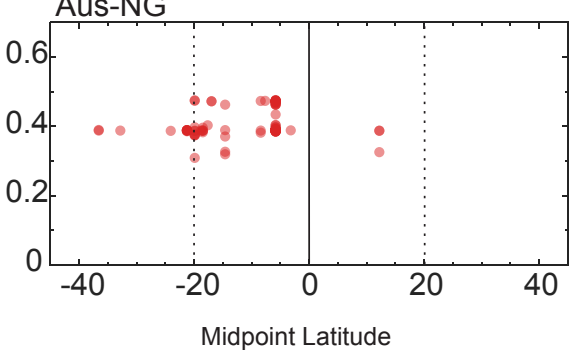

b)

All Pheidole

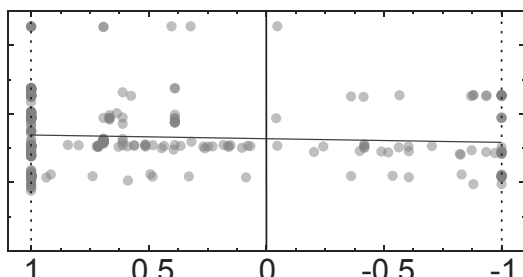

New World

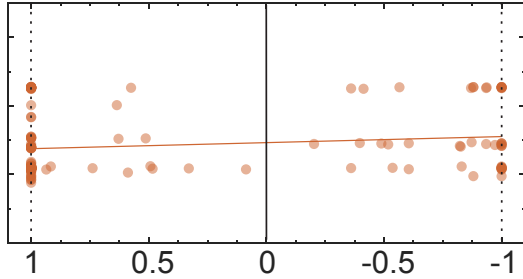

Asia-Africa

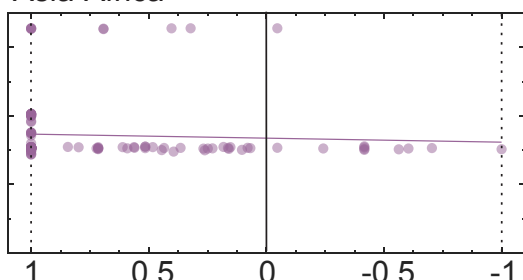

Aus-NG

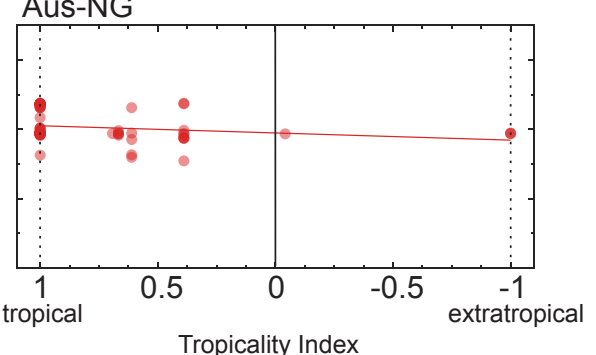

c)

All Pheidole
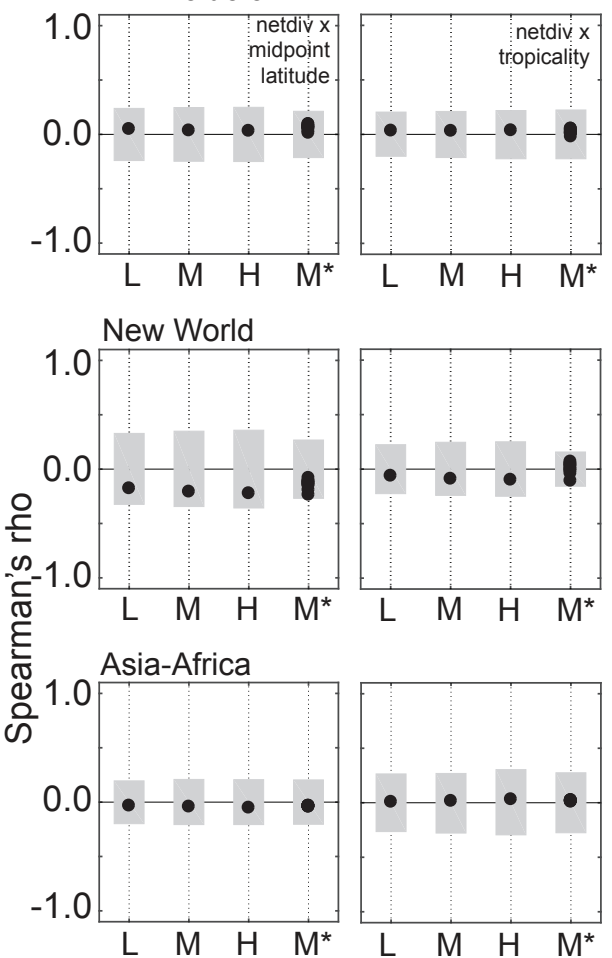

L M
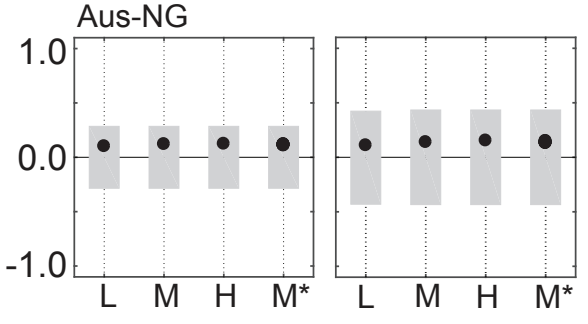

Figure 3: Net diversification rate inferred with BAMM as a function of latitude. Diversification rate of each Pheidole species (present day) inferred with BAMM using the "M" assumption of sampling completeness per species a) as a function of latitudinal midpoint and b) tropicality index, which varies from - 1 for a species with a range located competely outside the tropics to 1 for a species confined to the tropics. c) Spearman correlations (black dots) for net diversification and either absolute midpoint latitude (left) or tropicality (right), where the grey boxes reflect $95 \%$ null distribution generated with STRAPP. L, M, H, reflect different assumptions about unsampled species (low, medium, high estimates of total numbers of Pheidole), while $\mathrm{M}^{*}$ are 10 trees where temperate species have been culled to account for possible sampling bias (see methods). 
a)

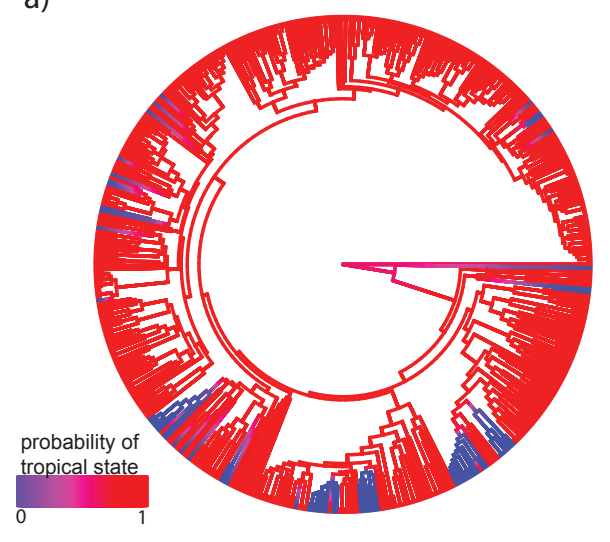

b)

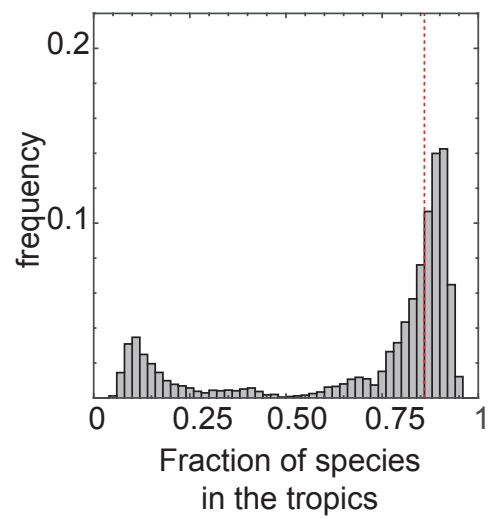

c)

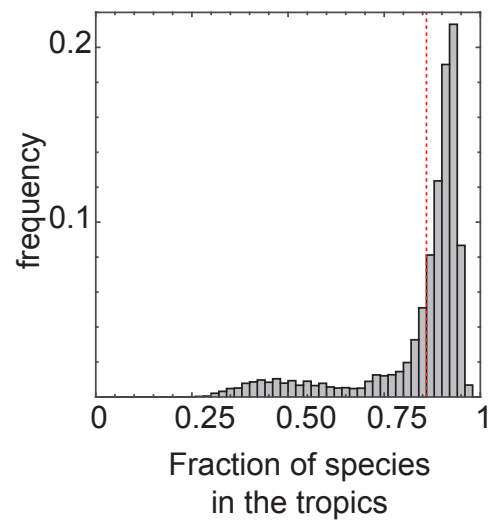

Figure 4 : Evolution of latitudinal affinity in Pheidole. a) Branch-wise probability of ancestral tropical state inferred from stochastic character mapping. b-c) Histograms of latitudinal richness differences beween tropics and extratropics simulated with stochastic character mapping on the empirical phylogeny assuming a tropical ancestor and the inferred degree of niche conservation using symmetric (b) or asymmetric (c) models of character evolution. The vertical dashed line is the empirical richness fraction. 